\title{
Changer de nom pour changer d'image. Le cas des modifications de dénomination de département
}

Rebranding under a new name. The case of modifying the names of local authorities

Cambiar de nombre para cambiar de imagen. El caso de las modificaciones de denominación del departamento

\section{Marie-Ange Grégory}

\section{(2) OpenEdition}

\section{Journals}

Édition électronique

URL : https://journals.openedition.org/mots/20466

DOI : $10.4000 /$ mots. 20466

ISBN : 15/11/2013

ISSN : 1960-6001

\section{Éditeur}

ENS Éditions

\section{Édition imprimée}

Date de publication : 15 novembre 2011

Pagination : 15-29

ISBN : 978-2-84788-326-8

ISSN : 0243-6450

\section{Référence électronique}

Marie-Ange Grégory, " Changer de nom pour changer d'image. Le cas des modifications de dénomination de département », Mots. Les langages du politique [En ligne], 97 | 2011, mis en ligne le 15 novembre 2013, consulté le 23 avril 2022. URL : http://journals.openedition.org/mots/20466 ; DOI : https://doi.org/10.4000/mots.20466 


\section{Changer de nom pour changer d'image. Le cas des modifications de dénomination de département}

26 février 1790 : l’Assemblée examine la dénomination des départements. La nouvelle division du royaume adoptée, Pinteville-Cernon ${ }^{1}$ propose, au nom du comité de Constitution, de nommer les quatre-vingt-trois départements constitués (Meuriot, 1917). La lecture des projets de dénomination s’interrompt devant les réclamations. Un vif débat s'ensuit. Les anciennes dénominations (provinciales) ne peuvent subsister; de même, l'appellation par le nom des chefs-lieux est rejetée. Toutes ces appellations sont suspectées de faire renaître «l'aristocratie ». La vivacité des échanges démontre déjà l’importance du nom des collectivités ainsi créées dans le nouvel univers de sens promu par la Révolution. Ainsi, pour Mirabeau, opposé à l'idée de simplement numéroter les départements et partisan de nouveaux noms : «Une dénomination fixe la raison». L'opération est finalement confiée au comité de Constitution qui s'inspire de la géographie physique au caractère par définition permanent : cours d'eau, montagnes, côtes, etc. La nomenclature est rendue officielle par lettres patentes du roi le 4 mars 1790. Les départements reçoivent alors, à quelques exceptions près, les noms qu'ils portent encore aujourd'hui. Depuis 1792, six départements ont changé de nom : la Charente-Inférieure a pris le nom de CharenteMaritime (loi du 4 septembre 1941), la Seine-Inférieure celui de Seine-Maritime (décret du 18 janvier 1955), la Loire-Inférieure est devenue Loire-Atlantique (décret du 9 mars 1957), les Basses-Pyrénées, Pyrénées-Atlantiques (décret du 10 octobre 1969), les Basses-Alpes s'appellent Alpes-de-Haute-Provence (décret du 13 avril 1970) et les Côtes-du-Nord, Côtes-d'Armor (décret n 90-201 du 27 février 1990). Ce sont ces exceptions, affirmations qu' " un toponyme peut être plus qu'un toponyme» (Boyer, 2008), qui constituent notre objet.

Si, en 1790, l'enjeu de la dénomination concerne surtout la reconnaissance d'une identité géographique, au $20^{\mathrm{e}}$ siècle, le processus de redénomination d'un département (de la circonscription administrative, de la collectivité

1. De Pinteville, baron de Cernon, député de la noblesse pour le baillage de Châlons-sur-Marne.

Université Nice-Sophia Antipolis (ERMES)

Marie-Ange.GREGORY@unice.fr

Mots. Les langages du politique $\mathrm{n}^{\circ} 97$ novembre $2011 \bullet 15$ 
territoriale et des administrés) s'avère un enjeu controversé mêlant notamment perspective économique et positionnement identitaire ${ }^{2}$. Pour autant, alors que l'attention portée par les élus locaux aux politiques d'identité et de communication s'est accrue, redonner une profondeur historique à ces procédés en s'intéressant aux changements de nom permet de relativiser la nouveauté des arguments et registres usités.

Le cas des redénominations de département s'avère stimulant à plusieurs égards. Tout d'abord, il s'agit d'une question sous-explorée. Parfois évoquées (Akin, 1999), les redénominations de département n'ont, à notre connaissance, jamais fait l'objet de recherches approfondies. Les monographies historiques n'y ont guère été plus sensibles, et les ouvrages de toponymie qui prennent pourtant fréquemment un cadre départemental ne s'intéressent qu'aux noms des communes. Ensuite, les six changements opérés reposent tous sur un changement d'image. Il ne s'agit pas d'une modification orthographique ou d'une volonté de différenciation (en cas d'homonymie), mais de la suppression de termes jugés péjoratifs (Inférieur, Bas, Nord). Alors que le Conseil d’État3 n'admet pas les demandes motivées par un but touristique ou économique, cet argument semble prédominant dans les discours pour faire valoir ce qui est présenté comme une «très légitime revendication». Enfin, l'échelonnement des changements de dénomination (de 1941 à 1990) et la longueur de la procédure (trente-et-un ans entre la première demande et le changement effectif pour les Côtes-d'Armor) offrent la possibilité de s'interroger sur le développement de la communication et des «politiques d'image » (Le Bart, 1999) au sein des collectivités territoriales.

La démarche proposée est d'autant plus heuristique que le développement des politiques de communication a suscité une réflexion sur les instruments, délaissant le premier marqueur qu'est le nom, et que les travaux des politistes s'interrogeant sur «la magie du nom » (Laurent, 1992) se sont davantage intéressés aux patronymes qu'aux toponymes. Il s'agit donc d'étudier cet enjeu controversé à travers les discours relatifs aux changements de nom des départements4, en particulier ceux tenus par les élus au sein des hémicycles locaux et nationaux ainsi que dans la presse locale. Appréhendant pourquoi et comment certains départements décident et réussissent à changer de nom,

2. Voir aussi dans ce dossier la contribution de Le Bart et Procureur, «Le nom du département comme problème. Entre logique d'attractivité et logique d'identité ».

3. L'article L3111-1 du Code général des collectivités territoriales dispose que «le changement de nom d'un département est décidé par décret en Conseil d’État sur la demande du conseil général».

4. Le matériau utilisé est issu de recherches menées aux archives départementales des Alpes-deHaute-Provence, de Charente-Maritime, des Côtes-d'Armor et de Seine-Maritime, nationales de Fontainebleau et de l'Assemblée nationale. Il est constitué pour l'essentiel de correspondances (cabinet du préfet, du président du conseil général), de brochures des syndicats d'initiative, de délibérations (conseils municipaux et généraux), de débats parlementaires, d'articles de presse, ainsi que de rapports du Conseil d’État. 
cette étude se propose d'examiner les enjeux de cette politique symbolique de redénomination. Sans constituer une analyse lexicale et discursive à proprement parler, l'étude porte avant tout sur la dimension subjective et évaluative des mots (perception par les usagers, réception par les milieux économiques et politiques, conflits et négociations entre les différents acteurs, etc.). Trois départements constituent principalement notre terrain : la Charente-Maritime (pionnière), les Alpes-de-Haute-Provence et les Côtes-d'Armor (les plus opiniâtres de par la récurrence de la demande).

\section{La volonté de redénomination : renverser le stigmate nominal}

\section{Inférieur, Bas, Nord: des mots aux maux}

Pour Georges Martin-Charpenel5, rédacteur d'un rapport sur la dénomination du département à la demande du préfet (1961), les critiques faites au toponyme Basses-Alpes sont de deux ordres:

1. Ces mots impliquent l'idée de monts peu élevés, de bordure du massif alpin à relief peu marqué. Les chaînes de montagnes de 2000 et $2500 \mathrm{~m}$ d'altitude étant fréquentes dans notre région où l'on trouve des sommets culminant à 3000 et $3410 \mathrm{~m}$, ils sont donc assez impropres pour la qualifier ou l'évoquer.

2. L'adjectif bas-basse a une indéniable valeur péjorative. Tout ce qui est bas, inférieur, paraît de moindre intérêt, de moindre qualité. Or, en particulier en matière de tourisme, dont on sait toute l'importance pour la survie de notre département, cet adjectif constitue un regrettable obstacle à éliminer.

Cet extrait cristallise les reproches le plus souvent formulés sur l'ensemble des départements sollicitant un changement de nom, à savoir l'inexactitude géographique et la valeur péjorative. Controversés, les qualificatifs Inférieur, Bas et Nord deviennent autant de motifs de changement ${ }^{6}$. Le nom, signe privilégié de l'identité, devient alors stigmate. Le "stigmate nominal», inspiré du cadre d'analyse d’Ervin Goffman, est «visible comme un trait physique, héréditaire comme une tare, codé comme un symbole et, de surcroît, cristallisé sur le signe le plus patent de l'identification individuelle et sociale » (Lapierre, 2006, p. 288).

L’inexactitude géographique révèle une erreur qui aurait été commise par les Constituants et qu'il convient de corriger. Dans les Côtes-du-Nord, bien que la démarche initiée vise avant tout à rayer un mot jugé « anti-touristique », priorité est donnée aux arguments géographiques. Lors d'une assemblée hôtelière à Perros-Guirec en 1953, Pierre Olivaux7 est invité par ses confrères à recueillir

5. Médecin, président de la Société scientifique et littéraire des Basses-Alpes.

6. Ce débat se retrouve au moment des discussions du projet de loi n 1413 portant réorganisation de la Corse (en deux départements) présenté à l'Assemblée nationale le 17 décembre 1974 .

7. Délégué hôtelier des Côtes-du-Nord au Comité d'études et de liaison des intérêts bretons (CELIB). 
l'avis des personnalités qualifiées du département sur un éventuel changement de nom. Suite à cette action, une motion est déposée lors de l'assemblée générale de l'Union départementale des syndicats d'initiative et de tourisme (UDSI) des Côtes-du-Nord en 1956. Reconduisant fidèlement cette motion, le vœu émis par le conseil municipal de Saint-Brieuc, le 10 juillet 1959, tendant à substituer, au nom du département des Côtes-du-Nord, celui de Côtes-d'Armor, débute ainsi :

Considérant que le nom du département des Côtes-du-Nord est manifestement impropre du point de vue géographique, ce département étant situé à l'ouest de la France,

Considérant que cette appellation est incorrecte, le mot « Nord» évoquant le froid, alors que le climat de la Bretagne, essentiellement doux et maritime, n'a rien de commun avec ce qu'il est convenu d'appeler un «climat nordique» [...].

Suit l'argument selon lequel le nom constitue un « élément nettement défavorable du point de vue touristique qui contrarie injustement les efforts de propagande des Syndicats d'Initiative », puis le rappel des précédents (CharenteMaritime, Seine-Maritime, Loire-Atlantique). Ce vœu briochin, qui privilégie le critère géographique, est ensuite repris par de nombreuses municipalités.

La valeur péjorative constitue un deuxième argument qui rassemble plus largement dès lors qu'il fait appel au groupe et à son identité. "Si “CharenteInférieure" signifie le cours inférieur de la Charente, le qualificatif prend, quoi qu'il en ait, un sens péjoratif regrettable », considère Paul Métadier, maire de Royan $^{8}$, conseiller général, pharmacien et principal acteur de la campagne pour le changement, dans une tribune intitulée «Il faut appeler notre département Charente-Maritime» (L'Indépendant, 27 août 1924). Le mot Inférieure serait une "dénomination malheureuse », notamment au point de vue de l'exportation à l'international. Le caractère péjoratif s'inscrit toujours dans un espace relationnel hiérarchisé, par exemple la Charente-Inférieure par rapport à la Charente.

À s'en tenir aux discours des promoteurs du changement de nom au moment où la contestation s'amplifie et s'intensifie, la connotation péjorative des qualificatifs Inférieure, Bas ou encore Nord relève de l'évidence. Une telle évidence s'avère pourtant trompeuse. En effet, dans la plupart des départements concernés, lorsque la question de la redénomination s'est posée pour la première fois, à des dates différentes, les responsables politiques n'y ont guère prêté attention et d'aucuns jugeaient l'idée saugrenue.

1. avril 1912 : le conseil général de Charente-Inférieure est réuni en séance.

8. Royan, qui s'est développée à la Belle Époque grâce à ses bains de mer, s'affirme dès les Années Folles comme une destination touristique renommée. À la veille de la seconde guerre mondiale, cette station balnéaire internationalement connue est fréquentée par de nombreux artistes et cinq cent mille baigneurs en été. 
La première commission donne lecture d'une lettre de Léopold Brugerolle, distillateur à Matha, adressée au président de la Chambre des députés, portant sur la « dénomination des départements de la Charente-Inférieure, de la Loire-Inférieure et de la Seine-Inférieure », accompagnée d'une pétition. Parcet envoi, Brugerolle sollicite l'appui de sa démarche par un vœu des conseillers généraux. Cette lettre contient la plupart des arguments utilisés par la suite.

Le voyageur de commerce qui vend à l'étranger des eaux-de-vie, des vins, du beurre et autres produits d'origine provenant de la Charente-Inférieure, se trouve fréquemment aux prises avec une difficulté sérieuse qui a pour cause le mot «Inférieure » du nom du Département producteur. [...]

Le mot «Inférieure» qui n'est qu'une indication géographique nécessaire pour l'acheminement de la correspondance, prend dans l'esprit des clients ignorant notre langue une tout autre signification, et ils s'imaginent qu'il s'agit de produits secondaires. [...]

Il est donc désirable pour les exportateurs de la Charente-Inférieure [...] qu'une modification soit apportée au nom de ce département qui pourrait s'appeler Charente-Maritime. [...]

Cette modification ne peut porter préjudice à personne. Elle ferait cesser immédiatement le discrédit créé par la confusion du nom géographique avec la valeur du produit.

Sans débat, le conseil général de Charente-Inférieure suit l'avis de la commission qui considère que « la substitution du mot "Maritime" au mot "Inférieure" n'est pas susceptible de donner plus de prestige à certains de [leurs] produits » et propose «le renvoi aux pouvoirs compétents " (conseil général de CharenteInférieure, 17 avril 1912). Dix ans plus tard, le conseil général est saisi d'une demande semblable émanant des membres du conseil municipal de Matha, réunis hors séance. Les considérations énoncées lors de la session d'avril 1912 demeurent. Le 25 avril 1922, les conseillers généraux se prononcent cette fois-ci - sans plus de débat - en faveur de la modification du nom du département, et décident d'engager des pourparlers avec leurs homologues de Loire-Inférieure et de Seine-Inférieure.

Les deux secours sollicités ne jugent pas alors devoir s'associer à la demande de la Charente-Inférieure, estimant sans intérêt pour eux le changement et lui préférant le statu quo (conseil général de Charente-Inférieure, 27 mai 1924). Leur réaction rejoint celle du conseil général de Charente-Inférieure en 1912. En effet, le rapport du docteur Fidelin, conseiller général de Seine-Inférieure, présenté en séance le $1^{\text {er }}$ octobre 1923, trahit un étonnement certain face à cette sollicitation inattendue qui a «donné l'impression d'une demande très naturelle jusqu'au paragraphe qui suggère des pourparlers avec les départements de la Loire-Inférieure et de la Seine-Inférieure ». Aucune objection n'est opposée au changement de dénomination de la Charente-Inférieure, bien au contraire : «La cause nous paraît tellement simple et juste qu'elle doit 
triompher, seule.» En revanche, l'éventualité d'une démarche collective les surprend. «Nous sommes conviés à formuler une demande semblable, et les trois requêtes seraient présentées ensemble au Parlement : cette union feraitelle une force? » Cette interrogation traduit bien que changer de nom ne peut être qu'une option individuelle et identitaire. Ce n'est pas le moment pour traiter ce sujet, tranche la deuxième commission, «sans s'expliquer davantage », au motif allusif qu' "il y a des nids de guêpes qui paraissent vides, et où il en reste d'engourdies » 9 . Dès lors, la commission "souhaite plein succès» à la Charente-Inférieure dans sa démarche «motivée par les raisons les plus dignes de déterminer gain de cause », mais rejette une requête analogue pour la SeineInférieure. La question du changement de dénomination de la Seine-Inférieure resurgit dans l'hémicycle départemental le 18 mai $1951^{10}$. Pour des raisons similaires au cas de la Charente-Inférieure, Georges Heuillard, conseiller général depuis 1937 et producteur de produits agricoles et laitiers, estime qu'«il devient évident que la collectivité est affublée d'une désignation péjorative».

Les différents exemples révèlent que l'origine des demandes se situe le plus souvent en dehors de la sphère politique. La mise sur agenda des redénominations résulte d'acteurs économiques, commerciaux ou touristiques. Le nom est péjoratif avant tout dans une perspective économique ; ce n'est qu'ensuite que les élites politiques s'en saisissent et convoquent le soutien de la population. Or, à partir du moment où une connotation péjorative est prêtée au département à travers son territoire, destination de vacances, et ses productions locales, l’idée s'étend par contagion à l'ensemble de la population et il est plus aisé de jouer sur une reconquête de la «face positive » (Goffman, 1973) de tout un département. Changer de nom permet alors d'effacer le stigmate et donc le discrédit, mais encore faut-il définir le nouveau nom qui donnera une image plus claire par rapport à sa localisation et plus positive.

\section{La recherche d'une dénomination adéquate}

«Côtes-d'Armor, c'est un joli nom.» Telle est la réponse du président de la République, François Mitterrand, donnée à Charles Josselin, dans un avion entre Tozeur et Tunis. Le président du conseil général des Côtes-du-Nord en est persuadé, «cet entretien a été déterminant » (Télégramme, 8 mars 1990). De même,

9. Cette prudence s'explique en partie par la volonté exprimée à plusieurs reprises de détacher l'arrondissement du Havre de la tutelle de Rouen et de créer un nouveau département. L'idée a notamment été présentée par Jules Siefried lors d'un meeting organisé le 25 mai 1879 au GrandThéâtre du Havre. Dans une motion votée à l'unanimité, « les soussignés, habitants de l'arrondissement du Havre, sollicitent du Gouvernement de la République et des Chambres la création du département de la Seine-Maritime».

10. À partir des années cinquante, le nombre de voyageurs augmente, y compris parmi les classes moyennes. La diffusion de l'économie du tourisme participe probablement de la multiplication des demandes de redénomination. 
vingt ans plus tôt, Raymond Marcellin, ministre de l'Intérieur, confie, lors d'une séance au Palais Bourbon, au député Marcel Massot : «L'appellation “Alpesde-Provence" est si jolie et si poétique que le Gouvernement vous accordera satisfaction» (20 novembre 1969). Le député la qualifie quant à lui de «beaucoup plus harmonieuse ». Un an plus tard, un décret officialise "Alpes-de-HauteProvence». «C'est aussi joli!», s'exclame le ministre (Assemblée nationale, 9 novembre 1970). Mais Marcel Massot ne partage pas cet avis : «L'appellation dont vous vantiez la poésie est alourdie. De toutes celles des départements français, elle est la seule qui soit composée de quatre mots.» Et il poursuit en déclarant que le conseil général des Alpes-de-Haute-Provence se voit dès lors « obligé d'entreprendre une nouvelle croisade » pour changer ce nom. La dialectique entre un nom actuel péjoratif et un nouveau nom « joli » est prégnante dans l'ensemble des discours tenus. Un nouveau nom participe de la construction d'une image de marque (Delattre, 2007). L'objectif est donc de se distinguer en portant un nom euphonique, mais aussi un nom inédit. De la sorte, les édiles se montrent attentifs aux autres usages du nom escompté. Par exemple, lorsque le conseil général des Basses-Alpes retient «Alpes-de-Provence», Maxime Javelly, conseiller général, réagit: « Mais nous allons avoir le nom d'une coopérative agricole?», faisant allusion à une grande coopérative basée à Manosque (conseil général, 12 décembre 1961). La volonté de se distinguer est encore plus forte entre départements. Ainsi, Georges Heuillard, conseiller général, note qu’il y a "déjà plusieurs départements “maritimes" » et, dès lors, que le département de Seine-Inférieure «n'est pas forcé de faire comme tout le monde » (conseil général, 18 mai 1951). De même, les élus de Loire-Inférieure énoncent leur préférence pour le qualificatif Atlantique, celui de Maritime étant «devenu presque aussi banal qu'Inférieur» (conseil général, 24 novembre 1955).

Bien évidemment, l'esthétique n'est pas le seul impératif à entrer en ligne de compte dans le choix d'une nouvelle dénomination. Tout d'abord, la loi encadre le changement de nom des collectivités locales, et une difficulté de plus pour les départements réside dans l'immatriculation alphanumérique ${ }^{11}$. À titre d'exemple, alors que le nom Haute-Provence est espéré par beaucoup, le directeur de l'Institut national de la statistique et des études économiques (INSEE) signale que cette dénomination changerait le numéro du département. Le conseil général se prononce alors pour la proposition Alpes-de-Provence, qui offre l'avantage de situer le département entre l'Allier et les Hautes-Alpes. De même, le pluriel s'impose aux Côtes-d'Armor pour figurer entre la Côte-d'Or et

11. La codification numérique, introduite à partir de 1922 pour l'immatriculation des plaques de voitures et des cartes de Sécurité sociale, s'est affirmée au fil du temps en repère dans l'inconscient collectif. Initialement abstraits de par leur attribution par ordre alphabétique à chaque département, ces numéros ont acquis une signification, dont le développement des produits dérivés, à l'instar de la marque « 64 », fournit une preuve. Aussi, les départements qui souhaitent changer de nom aspirent à conserver leur numéro. 
la Creuse. Au-delà d'une continuité souvent souhaitée, cette contrainte administrative explique donc que les changements ne soient que combinatoires, c'est-à-dire qu'une partie du nom soit conservée.

Ensuite, le nouveau nom doit mettre fin aux erreurs de géographie dénoncées et définir au mieux le département. Le promoteur de la Charente-Maritime, Paul Métadier, affirme ainsi que «presque aucun département n’est plus qualifié pour porter l'étiquette de “maritime” ». Arguant des «marins illustres» donnés par La Rochelle et Rochefort à l'histoire de la navigation, des «petits ports», des «stations balnéaires » qui prodiguent des «cures de climat marin », des «îles de Ré, d’Oléron, d’Aix», de «l'industrie maritime» (ostréiculture), de la «grande longueur sur la mer» ou encore de la «population maritime», il conclut : "Le mot “Maritime” est donc absolument logique; il s'impose » ( $L$ 'Indépendant, 27 août 1924). Dix ans plus tard, pour inviter les habitants du département à ne plus écrire que «Charente-Maritime», une affiche de propagande montre un trois-mâts où chaque voile gonflée par le vent est porteuse d'un des éléments évoqués (Le Phare de Royan, supplément du 18 février 1939). Le clinfoc met en avant un argument supplémentaire : «Un nom, c'est un programme d'action et de travail ». Ces éléments sont également repris durant cette période dans l'argumentaire des municipalités du département, les délibérations du conseil général ou encore les propositions de loi déposées par les députés.

Le changement opéré a donc une fonction de localisation : la mer borde la Charente-Inférieure, la Provence abrite les Basses-Alpes. Mais le changement peut aussi renvoyer à une communauté linguistique et culturelle. Grâce au qualificatif Armor, les Côtes se situent en Bretagne. Parmi les multiples appellations un temps envisagées (Côte d'Émeraude, Penthièvre, Rance et Trégor, Ajoncs d'Or), celles aux consonances bretonnes sont privilégiées : Côtes-d’Armor ou Côtes-d'Arvor. Dire que des côtes sont bordées par la mer n'apporte pas grande précision. Certains y ont d'ailleurs décelé une tautologie. La Société savante d'émulation des Côtes-du-Nord a fait observer, en effet, qu'en breton Ar Mor signifie « la mer » et que Côtes-d'Armor signifie donc "Côtes de la mer», et a émis le souhait qu'un nouveau qualificatif sans pléonasme soit trouvé, tel que Côtesdu-Norois ou Côtes-de-Granit (19 octobre 1959). Mais, plus que la signification, c'est bien la référence à l'Armorique qui est recherchée et renseigne les étrangers.

Enfin, le nouveau nom du département fait l'objet d'autant plus d'attention qu'il conditionne par là même le nom des habitants. Ainsi, la première fois que la transformation est discutée au sein de l'assemblée départementale des Basses-Alpes, Marcel Massot s’interroge : «Comment appellera-t-on dorénavant les habitants? Alpins de Provence ou Provençaux des Alpes? Pour ma part, né Bas-Alpin, j’aurais aimé mourir Bas-Alpin. » (conseil général, 12 décembre 1961) La même interrogation peut porter sur le nom des élus. Ainsi, André Bettencourt, jeune conseiller général, demande en souriant à Georges Heuillard, auteur de la proposition, s’il ne craint pas «que, désormais, les Conseillers 
Généraux de la "Porte Océane" soient traités de concierges " (conseil général, 18 mai 1951). En outre, les noms composés de département ne facilitent pas la création d'un gentilé et aucune règle n'existe en la matière. Les Bas-Alpins demeurent. En revanche, dès publication du décret, la presse baptise les habitants des Côtes-du-Nord en créant l'ethnonyme Costarmoricain.

La volonté ainsi exprimée de changer de nom apporte un nouvel exemple de ce que la psychanalyse qualifie de «force déterminante du nom » (Abraham, 1912), c'est-à-dire l'influence du nom sur le comportement de celui qui le porte. Changer de nom pour modifier son destin nécessite cependant de faire accepter le changement tant au niveau local que national.

\section{La négociation du changement de nom : accroître sa renommée}

\section{Les freins au changement}

Consulté pour la première fois sur un projet de changement de dénomination d'un département par les élus de Charente-Inférieure, le Conseil d'État a émis l'avis que «les noms des départements fixés depuis cent trente ans et entrés dans l'usage ne doivent être modifiés que pour de graves motifs et que la modification proposée en l'espèce ne se justifie par aucune raison d'ordre administratif » (dépêche du ministre de l'Intérieur, adressée au préfet de CharenteInférieure, 10 décembre 1924). Aucune publication émanant du Conseil d'État ne mentionne les critères intervenant dans l'examen des demandes de redénomination des collectivités territoriales. Les rapports du Conseil d'État offrent cependant quelques éclairages intéressants. Par exemple, dans une minute de note du 12 juin 1956, le rapporteur Hourticq juge «discutable» l'affirmation des élus de Loire-Inférieure selon laquelle « le qualificatif "Inférieure" ne peut que nuire à l'économie de la région» (conseil général, 24 novembre 1955). Néanmoins, rappelant les " précédents du département de la Charente-Maritime et de la Seine-Maritime», le rapporteur concède qu'il «semble difficile de renverser la vapeur». En outre, il considère que «si le principe du changement [est] admissible», c'est à la haute juridiction de "choisir». Or, commentant la préférence du conseil général pour l'adjectif Atlantique, il écrit : «C'est là qu'est le danger: nom publicitaire. Il y aura des départements d'azur ou d'émeraude.»

Ce scepticisme ne se cantonne pas au Palais-Royal et se retrouve aussi bien au ministère de l'Intérieur que dans certains articles de la presse nationale. Un exemple en est l'illustration de Dobritz parue dans Le Figaro (9 mars 1990). La vignette se scinde en deux, en miroir entre hier et aujourd'hui, tel un jeu des sept erreurs; sous un dessin à l'identique où une pluie battante domine, un seul détail change : le nom sur le panneau routier. Le même jour, les quotidiens 
locaux titrent quant à eux sur la « journée estivale » qui a accompagné la bonne nouvelle: «Qu'est-ce qu'il fait beau depuis qu'on est en Côtes-d'Armor!» (Télégramme, 9 mars 1990)

En outre, la longueur des procédures renseigne sur les réticences à accorder le changement de nom. Au-delà du principe d'immutabilité des noms, les raisons de refus révèlent la crainte de susciter des demandes émanant d'autres départements et la non-reconnaissance du caractère péjoratif avancé par les requérants. De la sorte, naît une dissonance entre les départements, impatients de porter un nouveau nom dans lequel ils placent tant d'espoirs, et le ministère de l'Intérieur qui freine la décision. Cette dissonance est d'autant plus forte que les départements ne comprennent pas en quoi leur « légitime requête » pourrait causer du tort. Plus encore, l'incompréhension réside dans la qualification de la demande par les deux parties. Pour le Conseil d'État, la stabilité des noms demeure la règle et le changement l'exception, comme pour les patronymes. Dès lors, le changement constitue une faveur que l'autorité peut refuser à son gré, une grâce. En revanche, pour les départements requérants, il s'agit d'un droit, voire même d'un dû. L'État doit corriger une injustice historique et faire disparaître une «inégalité de dénomination » dès lors que «toutes les parties du territoire français ont droit à la même sollicitude, à la même considération des pouvoirs publics» (proposition de loi du 12 mai 1939 tendant à donner au département de la Charente-Inférieure le nom de CharenteMaritime, présentée par James Sclafer et Amédée Delaunay, députés; exposé des motifs). Les départements s'érigent alors contre «l'inertie des pouvoirs centraux» et souhaitent «triompher de la routine administrative».

L'injustice nominale privant, aux dires des requérants, le département des subsides du tourisme, la contestation s'étend parfois sur le terrain financier. Ainsi, l'exposé des motifs de la proposition de loi du 11 mai 1939 tendant à substituer la dénomination «Charente-Maritime » au nom du département de la Charente-Inférieure, présentée par Roger Lefèvre, député, pointe les dépenses liées à la situation maritime du département : «lourdes charges» pour «la réfection et l'amélioration des ports», «liaisons entre les îles et le continent» ou encore «fonctionnement très onéreux de bacs automoteurs». Ces dépenses se traduisent par « des impôts lourds », et le député de conclure : «Un département qui s'impose régulièrement toutes ces charges a largement payé le droit de se prévaloir, dans son nom, de sa qualité de région maritime qui lui coûte si cher. » Par conséquent, Roger Lefèvre exhorte les parlementaires à modifier le nom de Charente-Inférieure afin de conjurer «la vertu maléfique d'un nom mal conçu» et de «cesser de pénaliser» le département.

Étonnamment, alors que les bénéfices escomptés - bien que difficilement chiffrables - sous-tendent la demande, le coût du changement est, lui, peu évoqué. La mesure est pourtant dispendieuse : remplacement du matériel de timbrage, modification de la signalisation routière, renouvellement des entêtes 
sur les courriers, etc. Seules les Côtes-du-Nord considèrent que les répercussions financières «doivent être soigneusement examinées » et instituent une commission d'étude chargée d'évaluer les conséquences du changement de nom (conseil général, 6 janvier 1960). Au vu du rapport, la dépense la plus importante serait supportée par l'administration postale, le renouvellement des griffes et timbres étant estimé à quinze mille nouveaux francs. Pourtant, en 1990, la dépense la plus importante est la communication : environ quatre millions de francs (soit deux fois plus que le budget habituel) sont consacrés à la promotion du département.

\section{La mobilisation entre promotion du territoire et quête d'identité}

Des oppositions existent aussi au niveau local mais, de faible portée, elles sont marginalisées par les défenseurs du projet. Ces derniers en sont conscients, le changement « est un résultat qui sera administrativement long à obtenir », «des années passeront» (Métadier, L'Indépendant, 27 août 1924). Mais le résultat n'est pas le seul enjeu de la mobilisation. II s'agit aussi d'unifier le territoire autour d'une identité commune et de faire connaître le département à travers un travail d'accompagnement visant à convaincre, tant à l'intérieur qu'à l'extérieur du département. Plusieurs procédés se mêlent, dans des proportions différentes dans chaque département, pour parvenir au(x) but(s).

Lors de sa séance du 27 mai 1924, le conseil général de Charente-Inférieure décide de maintenir sa délibération en vue de demander le changement de la dénomination (25 avril 1923) sans qu'il soit besoin d'aucune entente préalable avec les départements de Loire-Inférieure et Seine-Inférieure. Les communes du département sont alors consultées et plus de trois cents répondent favorablement (conseil général, 19 mai 1925). Quinze ans plus tard, à l'appel du député Roger Lefèvre, les conseils municipaux délibèrent de nouveau de mai à août 1939. La lecture des vœux municipaux révèle des similitudes, preuves de la coordination du mouvement, et seules quelques communes ne se prononcent pas ou s'opposent au projet.

Plus encore, dès 1924, Paul Métadier ${ }^{12}$ propose d'adopter la dénomination souhaitée, Charente-Maritime, sur les lettres, en-têtes et impressions. Progressivement, l'emploi du nouveau nom doit se généraliser pour que les pouvoirs publics n'aient plus qu'à consacrer par une loi l'usage établi. Cet appel est entendu par L'Indépendant de la Charente-Inférieure qui transforme son titre pour devenir, le 27 août 1924, L'Indépendant de la Charente-Maritime. Pas supplémentaire, en 1939, l'édile de Royan prend l'initiative de faire établir pour

12. L'édile royannais paraît précurseur des maires entrepreneurs tant il participe à l'attractivité de son territoire. Il fait, par exemple, de Royan une ville-étape du Tour de France en 1938 et offre chaque année, à ses frais, un feu d'artifice pour couronner la saison touristique. 
sa commune un cachet avec Charente-Maritime et sollicite de ses confrères une «approbation agissante». Autrement dit, Métadier encourage les maires à acheter un nouveau timbre humide, "dépense insignifiante», pour précéder et surtout accélérer la « régularisation » par les instances nationales (lettre parue dans Le Phare de Royan, 25 février 1939). Il aurait même «fait fabriquer, à ses frais, des sceaux disant “Charente-Maritime” » et en aurait « envoyé dans de nombreuses communes » (lettre du préfet au ministre de l'Intérieur, 27 mai 1939). Concomitamment, le ministre des Postes, Télégraphes et Téléphones déclare au député James Sclafer que les lettres portant l’indication CharenteMaritime seront normalement acheminées. Afin de rassurer les maires suite à une circulaire du préfet (24 mai 1939), Paul Métadier requiert l'avis d'Achille Mestre, professeur de droit à la Faculté de Paris, sur les conséquences administratives de la substitution ${ }^{13}$, dans les documents et sur les cachets officiels municipaux, de Charente-Maritime à la dénomination légale CharenteInférieure. Le juriste confirme que la «substitution ne saurait avoir d'effet en ce qui concerne la validité des actes administratifs » et ajoute que le terme Inférieure, à la « résonance invinciblement péjorative », lui paraît «particulièrement malencontreux». La consultation du professeur Mestre est communiquée aux agences de presse, accompagnée du commentaire : "La validité juridique du terme Charente-Maritime est reconnue». Pour une action plus efficace, Paul Métadier invite les maires du département à mettre en service partout le nouveau cachet à une date précise, le 15 avril 1939.

Alors que la presse est jusqu'alors restée discrète, l'initiative de substituer sur le timbre de la mairie de Royan «Charente-Maritime» à «CharenteInférieure » se voit consacrée très largement dans les journaux. Une brève revue de presse permet d'en apprécier la résonance. S'agissant de la presse parisienne, La Croix, Le Petit Parisien, Le Figaro, Les Échos dédient une chronique à cette action, et le Journal des Débats publie un feuilleton historique. La République salue "l'initiative révolutionnaire», L'F Fuvre loue "une victoire du bon sens et de l'esprit » et Paris-Soir (plus d'un million d'exemplaires) titre en page deux que Paul Métadier "agite l'étendard de la révolte». De même, L'Action française approuve «la Municipalité de Royan lassée par la torpeur du Ministère » et Le Jour évoque «les Charentais atlantiques qui ont franchi le Rubicon administratif». La presse régionale témoigne un semblable intérêt. L'OuestÉclair invite à repeindre les bornes kilométriques. La Dépêche Dauphinoise note que «toute “Inférieure” qu'elle est, la Charente... Maritime n’a pas eu jusqu’à présent à se plaindre du sort » puisqu'elle a fourni nombre d'hommes d’État. Bien évidemment, la presse locale couvre l’événement, mais déforme quelque peu l’information : «Sans plus attendre la décision administrative,

13. Plus largement, littérateurs et érudits sont mobilisés dans chaque département pour légitimer la demande et en accroître l'empan historique. 
les Maires de la Charente-Inférieure ont décidé de modifier les cachets municipaux qui porteront désormais : Charente-Maritime. » Paul Métadier fait à deux reprises la une du Cri de Royan, représenté tour à tour en statue de la Liberté, brandissant le nouveau cachet communal qui rayonne sur l'océan (mars 1939), puis en prêtre célébrant le baptême de la Charente-Maritime (mai 1939). L'enthousiasme se lit aussi dans la presse professionnelle : La Revue des Vins et Liqueurs, Europe-Colonies (commerce des céréales), L'Union des Épiciers, Le Jeu (organe des casinos) ou encore La Gazette des Pharmaciens, qui salue la verve de leur confrère. Les journaux étrangers (Belgique, Tunisie) applaudissent également l'audacieuse initiative. Ce coup médiatique offre une publicité gratuite au département et, plus encore, permet de consacrer l'emploi, dans la presse, de Charente-Maritime. Par exemple, Le Petit Parisien titre "La CharenteMaritime, paradis des petits réfugiés espagnols", et Le Populaire, "Congrès de la Fédération Socialiste de la Charente-Maritime».

Bien que la décision d'autoriser le changement revienne à partir de $1945 \mathrm{au}$ Conseil d'État, les hémicycles parlementaires constituent une scène supplémentaire pour l'action. Par exemple, le député Marcel Massot interpelle régulièrement, chaque année entre 1967 et 1970, en séance le gouvernement. Premier réveil adressé par l'élu au secrétaire d'État à l'Intérieur, André Bord, le «dossier [des Basses-Alpes] sommeille depuis deux ans dans les cartons de votre ministère» (Assemblée nationale, 6 novembre 1967). Le député se plaît à rappeler systématiquement les faits et, plus encore, à rappeler ses engagements au ministre. Que le sujet soit évoqué seul ou pour clore une intervention, l'interaction directe à la tribune pèse bien davantage qu'un courrier; d'autant plus que le député de l'opposition, applaudi sur les bancs de la Fédération de la gauche démocrate et socialiste et du groupe communiste, n’hésite pas à interpeller le gouvernement pour voir «consacrée cette appellation tant désirée et trop longtemps attendue » : «Il faudrait en finir, Monsieur le ministre, avec cette question irritante et quelque peu exaspérante pour la population de mon département qui attend depuis six ans.» (14 novembre 1968). Autre exemple : alors qu'un décret officialise les Pyrénées-Atlantiques, Marcel Massot use de la prétérition : "Je ne ferai pas au Gouvernement l'injure de penser qu'un traitement inégal serait fait à deux départements, selon que leurs représentants siègent dans la majorité ou dans l'opposition ${ }^{14}$. » (20 novembre 1969) À l'inverse, les membres du gouvernement peu diserts fournissent des réponses toujours mesurées : "la dernière phase de la procédure est engagée », « délai assez rapide» (6 novembre 1967), « désir sincère de donner satisfaction » (14 novembre 1968), «le dossier a été repris» (20 novembre 1969), etc.

14. Jean-Louis Tinaud, président du conseil général de Basses-Pyrénées et sénateur en 1962, lorsque l'assemblée départementale émet le vœu de s'appeler «Pyrénées-Atlantiques », obtient le changement de nom du département en octobre 1969, soit quelques mois après son entrée au gouvernement en tant que secrétaire d’État. 
Enfin, premiers concernés par le changement, les acteurs économiques et plus spécifiquement les professionnels du tourisme, constituent d'importants agents de mobilisation. À l'origine de la mise sur agenda des changements de nom, ils défendent par la suite avec ardeur le projet. En Côtes-du-Nord, le principal promoteur du projet est Alphonse Boulbain, président de l'UDSI du département en 1959, lors de la première grande offensive pour le changement : il sera surnommé «Papy Armor» par la presse en 1990. Entre-temps, il accumule tous les documents sur le sujet, agit aux niveaux local et national par une importante correspondance et surtout entreprend nombre d'actions. Deux plaquettes sont ainsi réalisées par l'UDSI : Un tour d'horizon objectifsur le changement de nom (1959, 16 p., diffusée à 30000 exemplaires) et Vers le changement de nom du Département des Côtes-du-Nord (1960, 15 p.). Alors que déjà 307 des 385 communes, au cours des années 1959 et 1960, ont adopté des vœux favorables au projet, la seconde plaquette publie en dernière page un vœu type et invite les conseils municipaux qui ne l'auraient pas encore fait à le remplir. Autre initiative : en 1962 est lancée une campagne publicitaire destinée aux lecteurs des grands périodiques et quotidiens d'Outre-Manche, vantant un département qui bénéficie du Gulf Stream : «In Britanny choose Côtes-d'Armor».

L'officialisation du changement de nom se vit différemment selon les situations. Après dix-huit ans d'efforts, la Charente-Inférieure devient Maritime à Vichy en 1941, et le souvenir de cette époque troublée n'incitera pas à commémorer l'événement. Après huit ans de procédure, les Alpes-de-Haute-Provence sont célébrées sans faste. Après trente-et-un ans d'espoir, une boussole indiquant l'ouest annonce dans la presse que « les Côtes-du-Nord ont perdu le Nord pour devenir les Côtes-d'Armor». En 1990, le conseil général célèbre à la fois le bicentenaire des départements et «l'an I des Côtes-d’Armor» (Magazine 22). Le «dernier-né très attendu » bénéficie d’un important plan médias (spots publicitaires, presse, autocollants, etc.). Premier président, premier préfet et premiers bébés des Côtes-d'Armor incarnent la «seconde naissance » du département. En 2000, les enfants nés le jour du changement sont invités à souffler leurs bougies au conseil général. En 2010, les jeunes nés dans le département en 1990 sont à l'honneur et se voient proposer un programme culturel.

\section{Références}

Abraham Karl, 1912, "La force déterminante du nom », Fuvres complètes, t. I, Paris, Payot, 1973, p. 114-115.

AkıN Salih éd., 1999, Noms et re-noms. La dénomination des personnes, des populations, des langues et des territoires, Rouen, Publications de l'Université de Rouen. BOYER Henri, 2008, «Fonctionnements sociolinguistiques de la dénomination topony- 
mique », Mots. Les langages du politique, $\mathrm{n}^{\circ} 86$, Toponymes. Instruments et enjeux, p. 9-21.

DelATTRE Éric, 2007, «Le changement de nom des communes françaises. Aspects économiques, marketing et stratégiques», Revue d'économie régionale \& urbaine, no 2, p. 269-291.

GoffmAn Erving, 1973, La mise en scène de la vie quotidienne, t. Il, Les relations en public, Paris, Minuit.

LAPIERRE Nicole, 2006, Changer de nom, Paris, Gallimard.

LAURENT Annie, 1992, "La magie du nom. Auto-regards des héritiers d'aujourd'hui. Les conseillers généraux dans le Nord-Pas-de-Calais depuis 1958 », L'hérédité en politique, J.-L. Parodi, C. Patriat éd., Paris, Economica, p. 173-186.

LE BART Christian, 1999, «Les politiques d'image. Entre marketing territorial et identité locale», Les nouvelles politiques locales, R. Balme, A. Faure, A. Mabileau éd., Paris, Presses de Sciences Po, p. 415-427.

Meuriot Paul, 1917, Pourquoi et comment furent dénommées nos circonscriptions départementales, Paris, Auguste Picard. 